\title{
Simplified Inelastic Acoustic-Phonon Hole Scattering Model for Silicon
}

\author{
F. M. Bufler, A. Schenk, and W. Fichtner \\ Institut für Integrierte Systeme, ETH Zürich, CH-8092 Zürich, Switzerland
}

\begin{abstract}
A simplified model for inelastic acoustic phonon scattering of holes in silicon is developed. It consists in approximating both the acoustic phonon energy and the square of the phonon wave vector by lattice-temperature dependent constants. The resulting scattering rate depends only on energy and thus facilitates the search of after-scattering-states during full-band Monte Carlo simulation. The simulation results for the velocity-field characteristics accurately agree with the experimental data at different lattice temperatures, while the population of hot-hole states is significantly enhanced compared to the elastic equipartition approximation. The value of the energy relaxation time to be used in hydrodynamic device simulations is roughly $0.1 \mathrm{ps}$.
\end{abstract}

\section{Introduction}

Modeling for TCAD applications is always a trade-off between computational speed and the accuracy of the models employed. In the context of full-band Monte Carlo (FBMC) device simulation, the elastic equipartition approximation [8] for acoustic phonon scattering has proved very useful. On the one hand, the scattering rate then depends on energy only and not on the wave vector, thus facilitating the search of the state after scattering. In addition, this scattering model allows for an exact computation of the Ohmic drift mobility via the microscopic relaxation time which requires only a one-dimensional integration over energy [2]. On the other hand, this elastic model is also able to accurately reproduce experimental drift mobility or velocity data both in the linear $[2,7]$ and in the nonlinear regime $[1,5]$ over a wide range of lattice temperatures. Nevertheless, the elastic approximation significantly underestimates the number of hot holes as has recently been shown [6] by a comparison with a model which includes inelastic acoustic phonons and yields - after recalibration of the acoustic and the optical coupling constants - similar good agreement with the experimental velocity data as the elastic model. It is therefore the aim of this paper to propose a new model for acoustic phonon scattering which combines the energy dissipation of the wave-vector dependent inelastic acoustic model with the simplicity of the elastic model while preserving the good agreement with the experimental drift velocity data. 


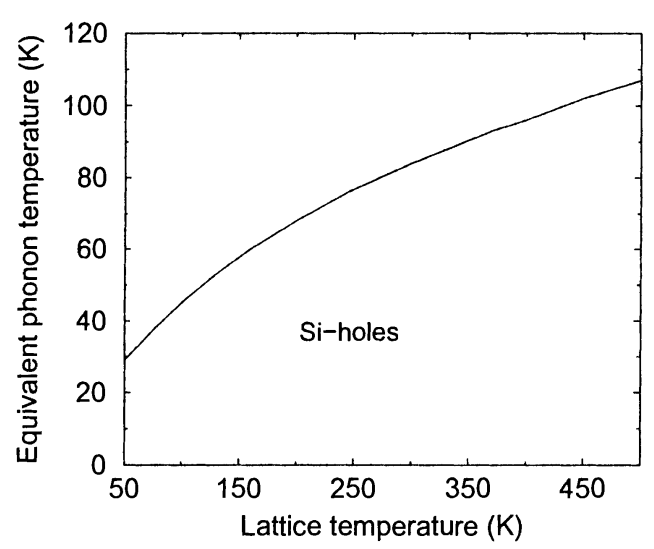

Fig 1: Mean acoustic phonon energy expressed in terms of the equivalent phonon temperature defined via $k_{B} \theta \equiv\langle\hbar \omega(q)\rangle$.

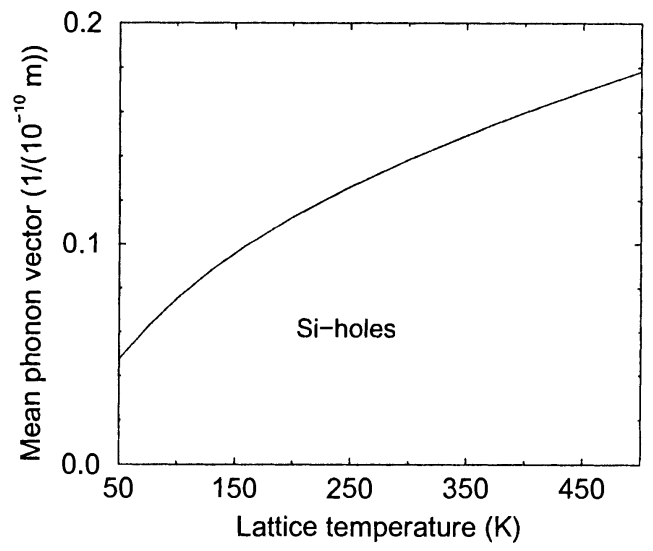

Fig 2: Modulus of the phonon wave vector transferred during acoustic phononhole scattering determined via $\sqrt{\left\langle q^{2}\right\rangle}$.

\section{Model}

The starting point is the scattering rate which can be written in the form (e.g. [6])

$$
S(\mathbf{k})=\sum_{\mathbf{k}^{\prime}} \frac{\pi}{\rho \omega(q)} \Delta^{2}(q)\left(N_{q}+\frac{1}{2} \mp \frac{1}{2}\right) \frac{1}{V} \delta\left(\epsilon\left(\mathbf{k}^{\prime}\right)-\epsilon(\mathbf{k}) \mp \hbar \omega(q)\right)
$$

where the overlap integral has been set to unity and the phonon wave vector q results from wave vector conservation. For optical phonons, the coupling constant $\Delta(q)=$ $D_{t} K$ and the phonon energy $\hbar \omega(q)=\hbar \omega_{\text {opt }} \equiv k_{B} \theta_{\text {opt }}$ can be approximated by constants with an equivalent phonon temperature of $\theta_{\mathrm{opt}}=731 \mathrm{~K}$. The inelastic acoustic phonons are characterized in the isotropic approximation via $\Delta(q)=\mathcal{E} q$ and an analytical formula for the phonon dispersion $\omega(q)$ as given in Ref. [6]. Instead of keeping the wave-vector dependent acoustic phonon model or applying the elastic equipartition approximation, our new approach consists of replacing in the above scattering rate both $\omega(q)$ and $q^{2}$ by constants which are determined by an adequate averaging procedure. For this, first the modulus of the phonon wave vector, $q=\| \mathbf{k}^{\prime}-$ $\mathbf{k} \|$, is averaged over a sphere in the spirit of an isotropic, parabolic band structure. This yields $q=\frac{4}{3} k$. Then the mean phonon energy, which is absorbed or emitted by a hole with wave vector $\mathbf{k}$, is obtained via an average with the hole distribution function. Approximating further the distribution function by the Maxwell-Boltzmann distribution leads with $\beta=1 /\left(k_{B} T\right)$ to

$$
k_{B} \theta_{\mathrm{ac}} \equiv \hbar \omega_{\mathrm{ac}}=\frac{\int d \epsilon \hbar \omega\left(\frac{4}{3} k(\epsilon)\right) \mathcal{D}(\epsilon) e^{-\beta \epsilon}}{\int d \epsilon \mathcal{D}(\epsilon) e^{-\beta \epsilon}}
$$

with the density of states $\mathcal{D}(\epsilon)$ and $k(\epsilon)=\sqrt{2 m(\epsilon)} \epsilon / \hbar$. The effective mass $m(\epsilon)$ is taken to be the energy-dependent effective density-of-states mass and thereby the main full-band-structure dependence is retained. As a consequence of this procedure, the mean acoustic phonon energy depends on the lattice temperature $T$ which is shown in Fig. 1. The same averaging procedure is applied to $q^{2}$ and the result is displayed in Fig. 2. The similar lattice-temperature dependence of $\sqrt{\left\langle q^{2}\right\rangle}$ indicates that the nonlinear part of the phonon dispersion $\omega(q)$ is negligible. 


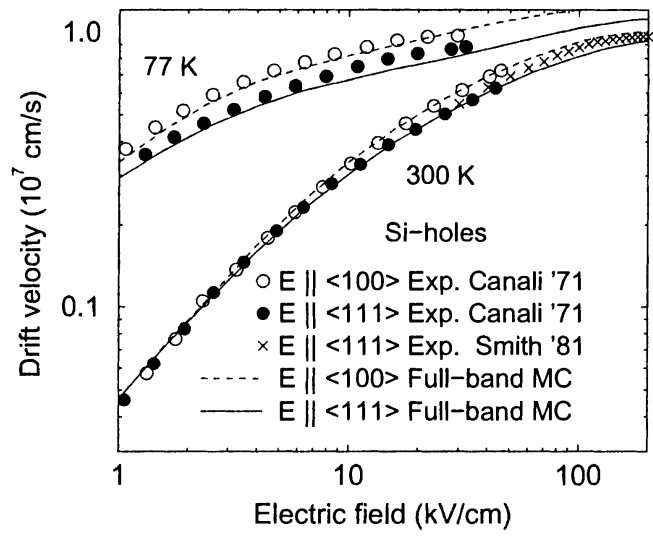

Fig 3: Results of the simplified inelastic model for the velocity-field characteristics of holes in Si at 77 and $300 \mathrm{~K}$ in comparison to experimental results $[4,10]$

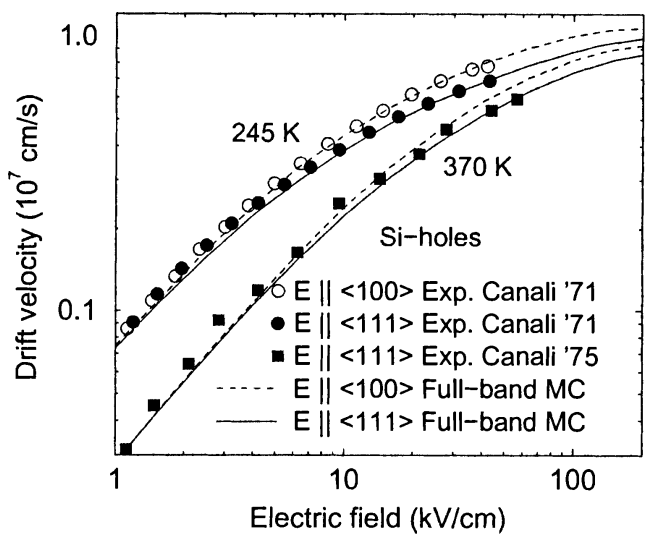

Fig 4: Results of the simplified inelastic model for the velocity-field characteristics of holes in $\mathrm{Si}$ at 245 and $370 \mathrm{~K}$ in comparison to experimental results $[4,3]$

\section{Results}

For this inelastic acoustic phonon model, the two coupling constants have been recalibrated with the result $\mathcal{E}=5.03 \mathrm{eV}$ and $D_{t} K=8.7 \mathrm{eV} / \AA$ (The equidistant mesh in $\mathbf{k}$-space for the full-band structure is $1 / 962 \pi / a$ where $a$ is the lattice constant). Note that the optical coupling constant is, in analogy to Refs. [7] and [6], smaller than in the corresponding model with elastic acoustic phonons [1,2]. Figures 3 and 4 demonstrate that the inelastic model with the new values for the coupling constants leads to a similar good agreement with the experimental velocity-field characteristics $[4,3,10]$ as the elastic model [1]. However, due to the smaller optical coupling constant, the population of high-energetic states by holes is significantly increased in the new model as is illustrated by the energy distributions in Fig. 5. This has, of course, corresponding consequences for the impact ionization coefficient and the substrate current in p-MOSFETs. It should be noticed that the number of high-energetic holes can still be increased by simply further reducing optical phonon scattering and readjusting the acoustic scattering rate. This would result, however, in a significant underestimation of both the experimental saturation velocity at high lattice temperatures and the experimental drift velocities in the low-field regime at low lattice temperatures [9]. Figure 6 finally reports the energy relaxation time for hydrodynamic device simulations where an increase from roughly 0.08 ps to 0.10 ps can be observed.

\section{Conclusions}

A new inelastic acoustic-phonon scattering model has been developed which (i) has only an energy-dependent scattering rate thus enabling an efficient search of afterscattering states, (ii) recovers the hot-hole tail of the energy distribution of more complicated inelastic models and (iii) accurately reproduces the experimental velocityfield characteristics over a wide range of lattice temperatures.

Acknowledgments This work was in part supported by the Kommission für Technologie und Innovation under Contract 4082.2. 


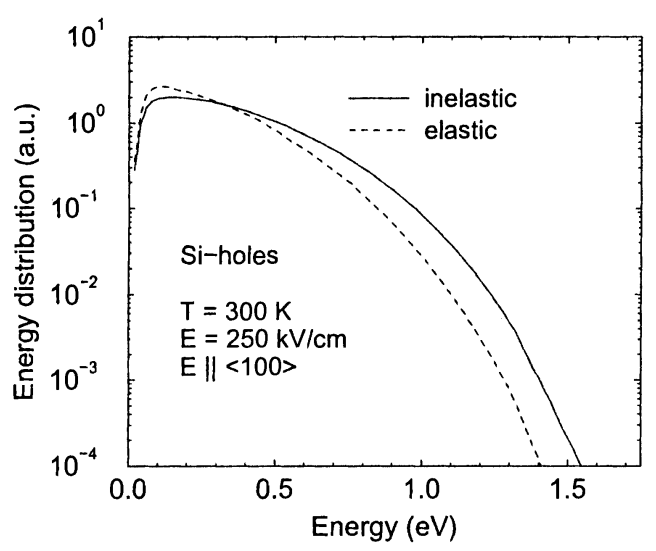

Fig 5: Energy distribution as a function of the hole energy $\epsilon(\mathbf{k})$ at $300 \mathrm{~K}$ for a field of $250 \mathrm{kV} / \mathrm{cm}$ in $\langle 100\rangle$ direction.

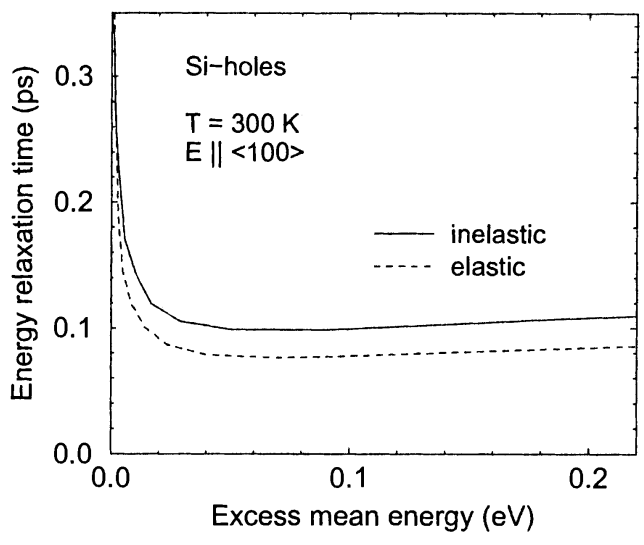

Fig 6: Energy relaxation time as a function of the excess mean energy $\langle\epsilon(\mathbf{k})\rangle-$ $\langle\epsilon(\mathbf{k})\rangle_{\mathrm{eq}}$ at $300 \mathrm{~K}$ in $\langle 100\rangle$ direction.

\section{References}

[1] F. M. Bufler, P. Graf, and B. Meinerzhagen. High-field hole transport in strained $\mathrm{Si}$ and SiGe by Monte Carlo simulation: Full band versus analytic band models. VLSI Design, 8:41-45, 1998.

[2] F. M. Bufler and B. Meinerzhagen. Hole transport in strained $\mathrm{Si}_{1-x} \mathrm{Ge}_{x}$ alloys on $\mathrm{Si}_{1-y} \mathrm{Ge}_{y}$ substrates. J. Appl. Phys., 84:5597-5602, 1998.

[3] C. Canali, G. Majni, R. Minder, and G. Ottaviani. Electron and hole drift velocity measurements in silicon and their empirical relation to electric field and temperature. IEEE Trans. Electron Devices, 22:1045-1047, 1975.

[4] C. Canali, G. Ottaviani, and A. Alberigi-Quaranta. Drift velocity of electrons and holes and associated anisotropic effects in silicon. J. Phys. Chem. Solids, 32:1707-1720, 1971.

[5] B. Fischer and K. R. Hofmann. Discretization of the Brillouin zone by an octree/Delaunay method with application to full-band Monte Carlo transport simulation. In Proc. SISPAD, pages 181-184, Leuven (Belgium), September 1998.

[6] B. Fischer and K. R. Hofmann. A full-band Monte Carlo model for the temperature dependence of electron and hole transport in silicon. Appl. Phys. Lett., $76: 583-585,2000$.

[7] M. V. Fischetti and S. E. Laux. Band structure, deformation potentials, and carrier mobility in strained Si, Ge and SiGe alloys. J. Appl. Phys., 80:2234-2252, 1996.

[8] C. Jacoboni and L. Reggiani. The Monte Carlo method for the solution of charge transport in semiconductors with application to covalent materials. Rev. Mod. Phys., 55:645-705, 1983.

[9] Y. Kamakura, I. Kawashima, K. Deguchi, and K. Taniguchi. Verification of hot hole scattering rates in silicon by quantum-yield experiment. J. Appl. Phys., $88: 5802-5809,2000$.

[10] P. M. Smith and J. Frey. High-field transport of holes in silicon. Appl. Phys. Lett., 39:332-333, 1981. 